\title{
Automatic Direction of Interactive Storytelling: Formalizing the Game Master Paradigm
}

\author{
Federico Peinado ${ }^{1}$ and Pablo Gervás ${ }^{2}$ \\ ${ }^{1}$ Departamento de Ingeniería del Software e Inteligencia Artificial \\ Universidad Complutense de Madrid, Spain \\ email@federicopeinado.com \\ ${ }^{2}$ Instituto de Tecnologías del Conocimiento \\ Universidad Complutense de Madrid, Spain \\ pgervas@sip.ucm.es
}

\begin{abstract}
During the fast evolution of automatic direction of Interactive Digital Storytelling many description of similar ideas have appeared in the academic literature. System architectures and workflows are usually presented using informal descriptions and diagrams, what makes difficult to identify what are the real contributions of each publication. In the way of establishing a safe ground for future research on this topic, this paper is a first step towards the formalization of a particular paradigm for developing automatic storytellers. The chosen paradigm is RolePlaying Game Mastering, which computational model is presented using UML.
\end{abstract}

\section{Introduction}

Specially during past decade, research on Interactive Digital Storytelling (IDS) has been growing relatively fast. Many papers about this topic are published each year in any of the conferences and journals that accept this kind of scientific contributions (from specific but also general fields such as Computer Entertainment and Education, Multimedia Systems, Artificial Intelligence, etc.). Some theoretical proposals have been described around the same idea: computational models for automatic control of an interactive narration.

The heart of the problem these research projects are trying to solve is a deep and well-known narratological question: the dilemma of Interactive Storytelling.

Authors of interactive narrations request some control over the plot that is unfolding in the computer of the user because they are the creators of the contents, the behaviours and the final purpose of the application; but at the same time interactors also need some control over the story they are enjoying because what motivate them is taking part in the plot as active agents and not only passive audience.

Several approaches are taken to develop an automatic direction system, and many different domains, environments and applications are used as test beds for illustrating the utility of those systems. Some of these approaches are becoming more common than others, what means they are recognized in the scientific community as valid paradigms for working in this field.

M. Cavazza and S. Donikian (Eds.): ICVS 2007, LNCS 4871, pp. 196-201, 2007.

(C) Springer-Verlag Berlin Heidelberg 2007 
In this paper we are presenting a preliminar formalization of a well-known theory, what we called the Game Master (GM) paradigm. This theory claims that the richer and more intuitive metaphor of an automatic director for IDS applications is a human GM controlling a role-playing game. This paradigm, sometimes too much overlooked, is a particular instance of the more general "centralized approach" to IDS management.

Because we usually found poorly detailed and informal figures in IDS research, probably because of the immaturity of the field, we propose the objective of improving and clarifying all the contributions that have appeared around te GM paradigm, describing them in a common and understandable framework.

The contents of the paper are organized as follow: Section 2 provides a short overview on IDS automatic direction related to the GM paradigm. Section 3 describes our proposal of a computational model for the system architecture and main workflow of this paradigm. Finally, Section 4 presents some preliminar conclusions of this research project.

\section{Background}

Widely different computational IDS approaches have been proposed in the area in which we can found those two big families of automatic direction approaches. There are proposals based on emergent behavior of non-player characters that achieve dramatic goals and approaches giving more responsibility to a central dramatic planning algorithm. Sometimes adding controllable NPCs to a centralized planner for achieving story goals or adopting a mixed approach, with a centralized director and a set of semiautonomous agents. There are also standalone dramatic planners that controls the most important narrative elements, like characters or the whole fictional world.

The idea of reusing concepts from Role Playing Games (RPGs) in the domain of IDS applications was there many years before it was published and more developed in recent research [1234]. Empirical studies, as the theory of Louchart and Aylett, or the categorization of Flowers, Magerko and Mishra, have been specially considered for the development of our model. Peinado and Gervás's translation of GM improvising rules to the world of computational problemsolving and the introduction of characters in the continuation of that model by Swartjes, Vromen and Bloom are also considered as a main reference in our proposal.

What is more difficult to find in the papers on automatic direction for IDS application are good formalization of computational models, workflows or software architectures that give support for this complex and knowledge-driven dynamic management. In order to start using a formal modeling language, we have chosen the Unified Modeling Language (UML) 5] for drawing a visual conceptualization of a GM-based system. This OMG standard is a well-known tool for modeling software applications, well documented and with very good development tools, so we consider it useful for modeling applications as the ones were mentioned before. 


\section{Modeling an Artificial Game Master}

In the context of this model, we make some assumptions about what an IDS system and an automatic director of an IDS system is.

First of all, we consider that an IDS system means "a computational system designed for software developers to build IDS applications" and IDS application means "a computational application designed for users (interactors) to take part in a concrete interactive experience, structured as a story represented in the computer". Sometimes behind an IDS application there is a more generic IDS system, sometimes not. In both cases it is possible to abstract the essential paradigm that researchers had in mind when designing their software, but usually for IDS application is more difficult.

Secondly, and automatic director for us means "a part of a computational system designed for the system itself to manage the experience of their potential users". This managements usually implies explicit representation of knowledge, intelligent mediation between authors and interactors and some goals or restrictions over the user experience, what it is called the multiform plot.

As we mentioned before, the Game Master paradigm is a particular case of the centralized architecture presented in many research projects. This architecture can accept some directed characters, what means they are in some way controlled by the director and therefore they have limited autonomy for developing the story. The game master can be implemented as a distributed system, even as a complex system with several components, but for control effects, only one narrative agent exists.

This section is dedicated to the computational model of the game master we formalize. There are some differences between this model and the ideas presented in other papers, but every decision is justified according to the different nature of a human GM and an "artificial" one, specially in the level of knowledge.

In figure 1 the UML component diagram of our proposal is shown. The model corresponds to a GM-based system architecture, i.e. a system with an automatic director of IDS applications following what we have called the Game Master paradigm.

In the center of the model there are two nodes: "Narrative Environment" and "Director". The first one is the virtual environment in which the narrative action is presented to the users (here called Interactors). It usually represents a 3D world with objects, locations, characters and every element needed to tell the story. Although using their imagination is also possible for the players, this environment restricts a lot that aspect of playing games. Players and environment have a good flow of information.

The second node replaces the human GM, being an artificial GM in the form of a software system. Both director and environment are communicated because they send and receive information, but in this case specific adapters are needed because interfaces for both nodes can be quite different due to the different nature of an AI program and a graphic or multimedia engine. Inside the Director there is a Knowledge Model and extensions for each domain of knowledge that the 


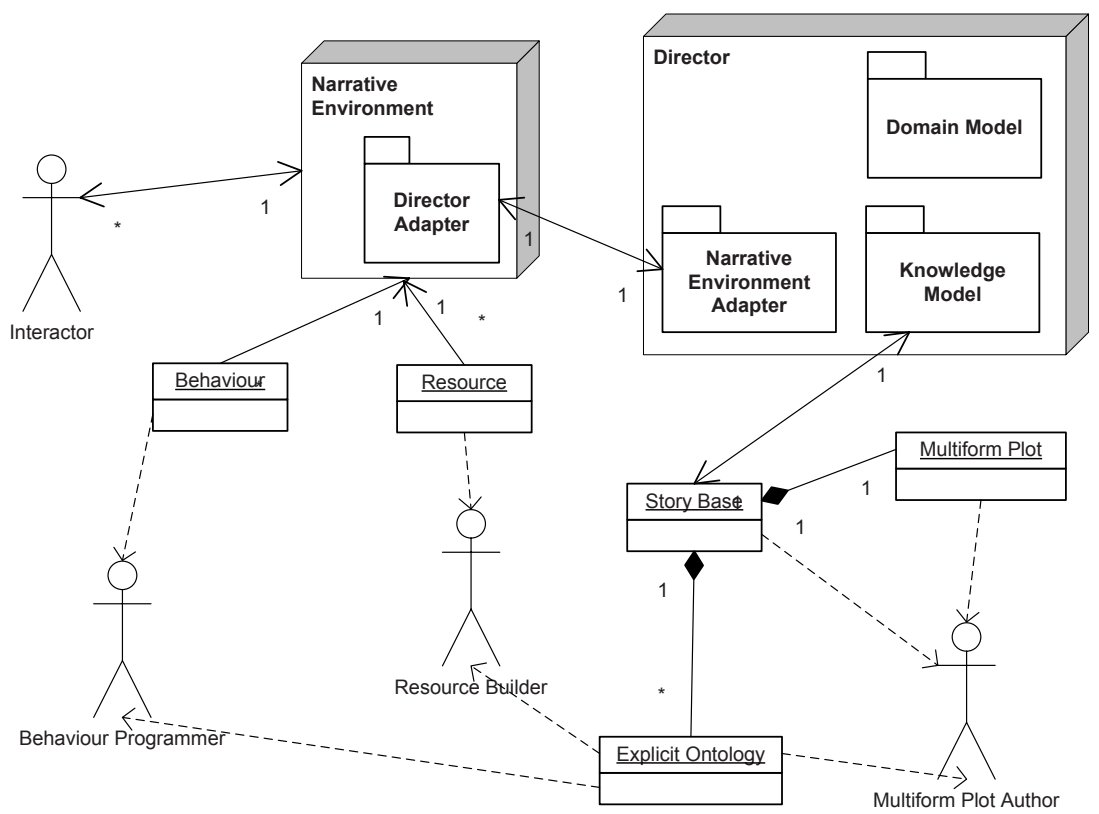

Fig. 1. A model of an automatic director inspired in a human Game Master

director needs in order to understand what is happening in the story and to intervene accordingly.

The Adventure, the plot draft that the human Game Master used to follow, it is called Multiform Plot in this model, developed also by an external Multiform Plot Author. This multiform plot is also the input for the environment, but because of the format it can be easily integrated in the Story Base and taken as part of the knowledge model of the director; so dependency with the Multiform Plot is implicit, but included in the model. The Story Base usually contains complete plots or some partial plots with elements that are useful in order to reuse old material when directing a new story.

Here clearly the Director is not the author of the multiform plot, but it can add some successful experiences to the Story Base, even in real time due to the capabilities a computer has and a human being has not.

Resource Builder and Behaviour Programmer are the two roles of the people involved in creating the material for the virtual environment. The game system must be ideally implicit in the environment, so Interactors can discover it at the same time they play. Director do not need to depende on those things because the communication with the environment is enough fluid to check that during each significant situation in the story.

An explicit representation of knowledge is needed, an Explicit Ontology, what is just a part of the knowledge the Interactors have about the fictional world, but enough for the Director to understand what happens in the story. In real 
life there is a strong game background and common sense assumptions shared by the GM and the players, but here all that knowledge is limited to the basic information about the game that can be coded in the ontology.

This model is being applied in the development of the centralized knowledgeintensive automatic director of the Knowledge-Intesive Interactive Digital Storytelling (KIIDS) system [6]. This director uses Case-Based Reasoning to model the kind of decisions a GM takes to create on the fly the next scene he is trying to introduce in the game.

\section{Conclusions}

In this paper we present a computational model of a human game master. It is designed as a centralized intelligent system, replacing the human GM, that perform some automatic control over the dynamic development of the story. Far beyond mediation, the system directs the story towards well defined goals using a multiform plot for that.

World and specially characters are not considered as truly narrative agents, just as non-completely autonomous extensions of the game master (the behaviours of the Narrative Environment represent those characters). Shared knowledge between players, GM, adventure authors and game system builders is replaced by an explicit ontology incorporated in the knowledge model of the Director. Data structures are unified in a story base, supporting multiform plots and complete stories. Communication interfaces between the Narrative Environment and the Director appears, because data flow now it is not transparent and fluid as players talking with the GM was.

The preliminar formalization presented here could be a step forward to the information interchange in our field. Although this paper has no room for other UML diagrams that describe low-level details of our model, the basic ideas have been presented.

The final goal is to make the community move towards better presentation of their ideas and results, using UML or other formal languages (maybe more appropiated for low-level description) to express, compare and evaluate different approaches, finding compatibilities and synergies, distributing the work in small parts, looking forward a better understanding of what IDS is and how it can be controlled by the computer.

\section{Acknowledgements}

This research is funded by Ministerio de Educación y Ciencia (TIN2006-14433C02-01 project), Universidad Complutense de Madrid and Dirección General de Universidades e Investigación de la Comunidad de Madrid (UCM-CAM-910494 research group grant). 


\section{References}

1. Louchart, S., Aylett, R.: Towards a narrative theory of virtual reality. Virtual Reality $7(1)(2003)$

2. Peinado, F., Gervás, P.: Transferring game mastering laws to interactive digital storytelling. In: Göbel, S., Spierling, U., Hoffmann, A., Iurgel, I., Schneider, O., Dechau, J., Feix, A. (eds.) TIDSE 2004. LNCS, vol. 3105, pp. 48-54. Springer, Heidelberg (2004)

3. Swartjes, I., Vromen, J., Bloom, N.: Narrative inspiration: Using case based problem solving to support emergent story generation. In: International Joint Workshop on Computational Creativity, London, UK (2007)

4. Flowers, A., Magerko, B., Mishra, P.: Gamemasters and interactive story: A categorization of storytelling techniques in live roleplaying. In: Futureplay, London, Ontario (2006)

5. Object-Management-Group: Unified modeling language. http://www.uml.org/

6. Peinado, F.: Knowledge-intensive interactive digital storytelling system. http://federicopeinado.com/projects/kiids/ 